\title{
Sleep improves when patients with chronic OA pain are managed with morning dosing of once a day extended-release morphine sulfate (AVINZA ${ }^{\circledR}$ ): Findings from a pilot study
}

\author{
Murray Rosenthal, DO \\ Polly Moore, PhD \\ Eric Groves, MD, PhD \\ Tad Iwan, BA \\ Lisa Greenberg Schlosser, MS \\ Zofia Dziewanowska, MD, PhD \\ Andres Negro-Vilar, MD, PhD
}

\section{ABSTRACT}

Study objective: To investigate the effect of once-aday extended release of morphine sulfate AVINZA $A^{\circledR}$ ( $A-M Q D$ ) on polysomnographic measures of sleep in a population of chronic osteoarthritic pain patients with sleep difficulties.

Design: Single-center, single-blind, placebo-lead-in, $30 \mathrm{mg}$ or $60 \mathrm{mg}$. Patients' sleep and neurocognition were objectively measured at a sleep laboratory, and patients self-rated their pain, sleep, and other functions.

Participants: Thirty-four participants (26 to 75 years old) complaining of sleep difficulties and chronic, stable pain secondary to hip or knee osteoarthritis.

Interventions: Participants had a screening visit on current pain medication and then, following a singleblind placebo run-in period, received $30 \mathrm{mg} / d$ of $A-M Q D$ for six days. At day 6, doses for participants with incomplete pain relief on the Brief-Pain-Inventory (BPI) pain scale were increased to $60 \mathrm{mg} / \mathrm{d}$. Treatment continued for another eight days at the new dose level (14 days for a subgroup at $60 \mathrm{mg} / \mathrm{d}$ ). Sleep was objectively measured by all-night polysomnography (PSG) at screening while on the participants' current pain therapy, at baseline following a placebo run-in and at the end of treatment while on A-MQD.

Outcome measures: PSG parameters evaluated included Total-Sleep-Time (TST), Wake-timeafter-SleepOnset (WASO), Sleep-Efficiency (SE), Latency-to-Persistent Sleep (LPS), Latency-to-REM-sleep, the Number-ofAwakenings (NAW), the time spent in each stage of sleep, and REM-sleep-latency. Subjective evaluations included participants' estimations of sleep time and sleep quality, the Epworth-Sleepiness-Scale (ESS), the BPI, and participant acceptance of and relief due to current therapy. Assessments of neurocognitive function were also made.

Results: Sleep initiation and maintenance tended to improve with A-MQD as demonstrated by the increases in TST and SE and decreases in WASO and NAW as compared with placebo-baseline values. Sleep architecture was preserved by the study drug and some increases in stage 2 and 3/4 sleep were seen compared with placebo baseline. Subjective ratings of sleep quality and sleep time were significantly improved with treatment, as were BPI scores and ratings of medication acceptance and pain relief. A-MQD was generally well tolerated.

Conclusions: $A-M Q D$ was an effective treatment for pain, and this study treatment was associated with improvement of both objective and subjective sleep parameters in participants with chronic osteoarthritic pain.

Key words: sleep, sleep quality, chronic pain, polysomnography, $A V I N Z A \circledast$ capsules

\section{INTRODUCTION}

Chronic pain is highly correlated with sleep disturbance. ${ }^{1-3}$ The incidence of disturbed sleep in participants with arthritis is estimated at nearly 60 percent. ${ }^{4-6}$ Importantly, baseline pain levels may even be predictive of sleep difficulties up to two years in the future. ${ }^{4}$ Furthermore, the relationship between disturbed sleep and pain appears to be bidirectional: pain worsens sleep difficulties and poor sleep heightens the perception of pain..$^{7-9}$ Increasing pain levels may be correlated with an increasing risk for insomnia, ${ }^{2}$ and recurrent poor sleep may be linked to muscular pain, tenderness, and fatigue. ${ }^{7,8}$ 


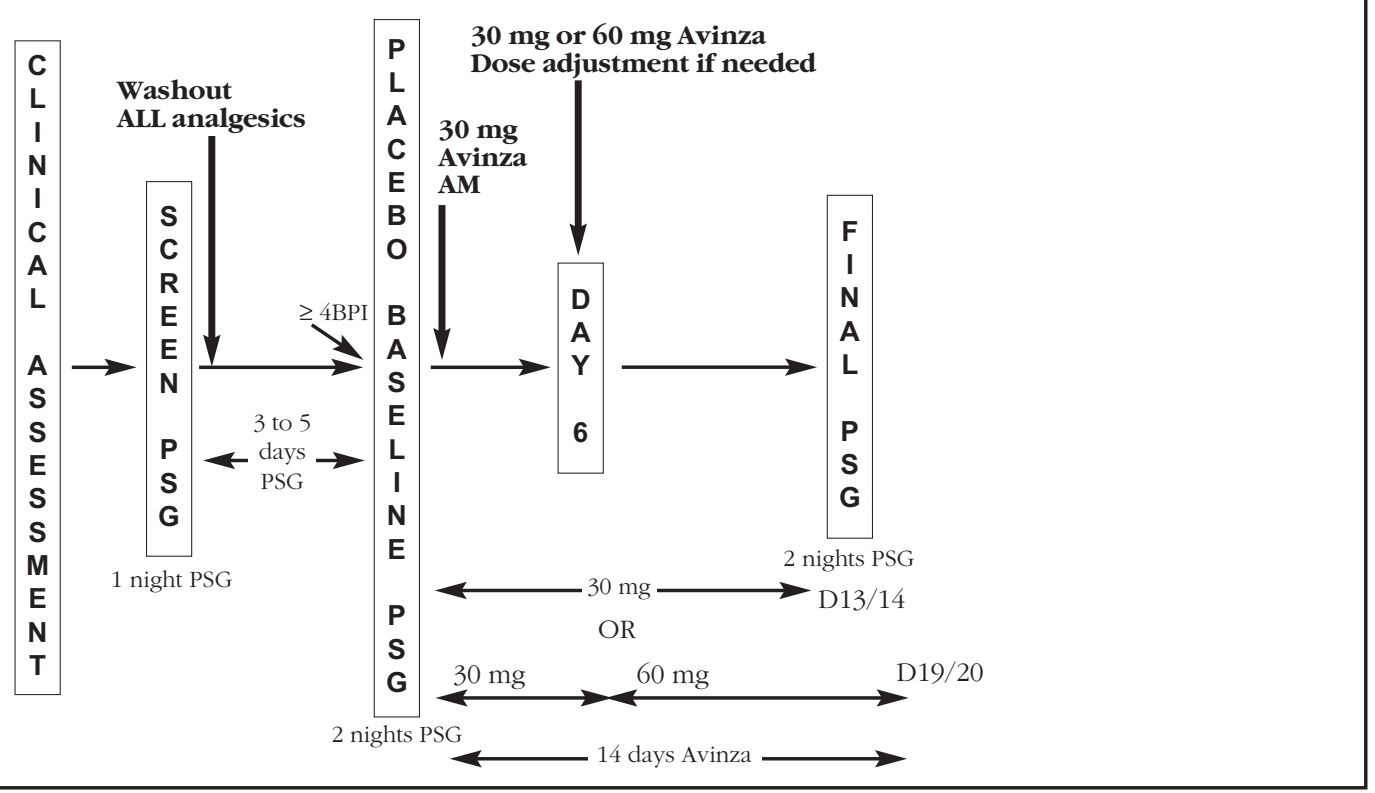

Figure 1. Study schema for analyzed subgroups.

Opioids are a commonly used pharmacological tool for the management of chronic to moderate to severe pain. Yet, there have been relatively few studies using objective sleep measurements to evaluate the effects of these medications on any participants, much less chronic pain participants. A publication of a limited study suggests that acute nighttime (presleep time) administration of opioids may suppress rapid eye movement (REM) sleep and slow wave sleep and may unexpectedly increase wakefulness. ${ }^{10}$ On the basis of this limited experience related to acute administration, it might be speculated that in chronic pain participants opioid medications given to alleviate pain could in fact be disruptive to sleep and could contribute to the participant's sleep disturbance, rather than ameliorating it. Studies by Caldwell et al., ${ }^{11}$ Rauck et al., ${ }^{12}$ and Panjabi et al. ${ }^{13,14}$ are in contrast to this, where osteoarthritis participants stated, in selfadministered questionnaires, that pain relief with opiates improved the quality of their sleep.

The use of sustained release opioids (SROs) may be responsible for the improvements in sleep as found by Caldwell, Rauck, and Punjabi. The pharmacokinetics and analgesic properties of a given SRO are highly dependent on the release profile of the drug delivery system. Therefore, two different modified-release formulations of the same opioid may yield significantly different profiles, even when administered in a similar manner.

Release of morphine sulfate (A-MQD) is a morphinebased SRO with a novel modified-release formulation specifically designed for once-daily dosing. ${ }^{11}$ The present exploratory study was designed to determine whether alleviation of chronic pain in osteoarthritic participants via A-MQD would produce concurrent improvements in polysomnographic (PSG) sleep measures in addition to confirming prior reports of subjective sleep improvement. Correlation of PSG findings with measures of neurocognition as well as quality of life measures was also assessed.

\section{METHODS}

\section{Study design}

This single-center, placebo-lead-in, single-treatment, single-blind study was conducted at a US site. Two doses (30 mg, titrated to $60 \mathrm{mg}$ as needed for a subset of the population) of A-MQD were evaluated in participants with documented osteoarthritis complaining of sleep disturbances secondary to their osteoarthritic pain. The protocol for this study was approved by an institutional review board, and the study was conducted in accordance with the Declaration of Helsinki and the Good Clinical Practice guidelines.

Participant selection involved three screening steps (Figure 1). First, potential participants underwent a clinical assessment visit. Second, participants returned for a polysomnographic screening visit (screening) while continuing the participants' then current pain therapy. Third, participants who continued to be eligible were then withdrawn from their current pain and sleep medication during a fiveday single-blind placebo-washout period that concluded with two nights of PSG evaluation (baseline). Subjective and neurocognitive evaluations were performed prior to discharge on the second morning (baseline for these evaluations). At this point, to continue to be eligible, participants had to have a score of $\geq 4$ on the Brief-Pain-Inventory (BPI).

Participants who passed these three screening evaluations were formally entered into the study and were then 
provided with 14 days of active treatment $(30 \mathrm{mg} / \mathrm{d} \mathrm{A}$ MQD). Participants returned to the clinic on the sixth day of active treatment to repeat the series of subjective and neurocognitive tests performed at placebo baseline. At this visit, the dose of A-MQD was increased to $60 \mathrm{mg} / \mathrm{d}$ for participants reporting inadequately responsive pain. Participants returned to the clinic on the thirteenth- and fourteenth-day of treatment for a final two nights of PSG evaluation (day 13/14). Again, the subjective and neurocognitive batteries were repeated and final safety evaluations were performed prior to the final discharge on day 15.

As this was a pilot study, part way through the study, an interim analysis was conducted to examine the internal consistency of the data. This analysis suggested (see discussion section) that the two treatment arms $(30 \mathrm{mg} / \mathrm{d}$ for 14 days vs $30 \mathrm{mg} / \mathrm{d}$ for six days followed by eight days at $60 \mathrm{mg} / \mathrm{d}$ ) did not represent comparable exposure to constant doses of study drug. At this point, participants whose dosage had been increased to $60 \mathrm{mg} / \mathrm{d}$ were provided with treatment at this dose level for a further full 14 days, thus spending a total of 20 days on study treatment. Participants in this last group did not repeat the day 6 assessments after six days at the new dose level and engaged in the final PSG evaluations on day 19 and 20 (equivalent to day 13/14 for the other groups) and the final subjective assessments on day 21 (equivalent to day 15 for the other groups).

Regardless of treatment arm, concomitant medication was restricted. Both prescription and OTC pain and sleep medications were prohibited. In addition, the use of any form of steroids and viscosupplementation in osteoarthritic joints was not allowed. Rescue medications for either pain or sleep were not allowed.

\section{Subject recruitment and selection}

Male and female participants with osteoarthritis at a hip and/or knee joint, aged 18 to 80 , in relatively good health were recruited for this study through local physicians and newspaper advertisements. Participants were eligible if they reported sleep disturbances secondary to their osteoarthritic pain and had been taking NSAIDs, acetaminophen, and/or a prn analgesic containing an opioid (maximum of $30 \mathrm{mg} / \mathrm{d}$ in morphine equivalents) for at least three months. Participants were further required to 1) not have a diagnosis of any chronic pain syndrome that would interfere with the assessment of osteoarthritis symptoms, 2) not have prior replacement surgery or other clinically significant disease at the affected joint(s), 3) not have a history of substance abuse or dependence, and 4) not receive any steroids within 30 days prior to baseline assessments, intra-articular steroids in within 60 days prior to study baseline, or intra-articular viscosupplementation at the affected joint(s) within six months of the start of the study.
After signing an informed consent statement, prospective study participants underwent a screening process that included a physical examination, clinical laboratory tests, and a 12-lead ECG. Qualified participants were invited to undergo a PSG screening night while continuing their current pain therapy. Participants who met the screening criteria for other sleep disorders (i.e., sleep apnea, periodic limb movement disorder) during the PSG screening night were no longer eligible for participation.

Next, eligible participants underwent a five-day placebo washout from all pain and sleep medications. At this point (placebo baseline), participants were finally invited to enter into a single-blind treatment if they had an average pain rating of $\geq 4$ on the BPI scale.

\section{Study procedures}

Study drug. A-MQD (30 and $60 \mathrm{mg} / \mathrm{d}$ ) was evaluated in this study. Medication $(30 \mathrm{mg} / \mathrm{d})$ was dispensed to participants after a single-blind placebo-baseline PSG night followed by next-morning neurocognitive, subjective pain, and sleep assessments. Participants were educated on the possible side effects of the study medication and cautioned about drowsiness while driving. They were then instructed to swallow one capsule of study medication in the morning each day for the next 14 days. Participants complaining of inadequate pain relief were titrated to $60 \mathrm{mg} / \mathrm{d}$ at the day 6 visit.

Polysomnography. PSG recordings were performed by experienced technicians and were scored according to the methodology of Rechtschaffen and Kales ${ }^{15}$ by a single registered polysomnographic technologist. Scoring was later confirmed by a blinded external, accredited polysomnographer. Recordings were taken in the sleep laboratory at screening (day -6, 5), baseline (day $-2,-1$ ), and during treatment (day 13 , 14) for a period of 480 minutes with lights out beginning at the participant's habitual bedtime. PSG values are the mean of each two-night period.

Subjective assessments. Participants completed several subjective assessments of their sleep and pain. These evaluations were completed at screening (day -5), baseline (day 0), and during treatment (day 6,15) and included the Epworth Sleepiness Scale (ESS), a sleep quality questionnaire, the Brief Pain Inventory (BPI), and ratings of the acceptability of and relief provided by their current therapy. The ESS is an eight-question instrument that assesses the participant's subjective sense of the likelihood of falling asleep in various real-life situations. Each question is rated on a scale of 0 ("would never doze") to 3 ("high chance of dozing"). The sleep quality questionnaire asked participants to rate their quality of sleep on a VAS scale of 0 to 100 and to estimate the average number of hours of sleep they were getting per night. The BPI is a 


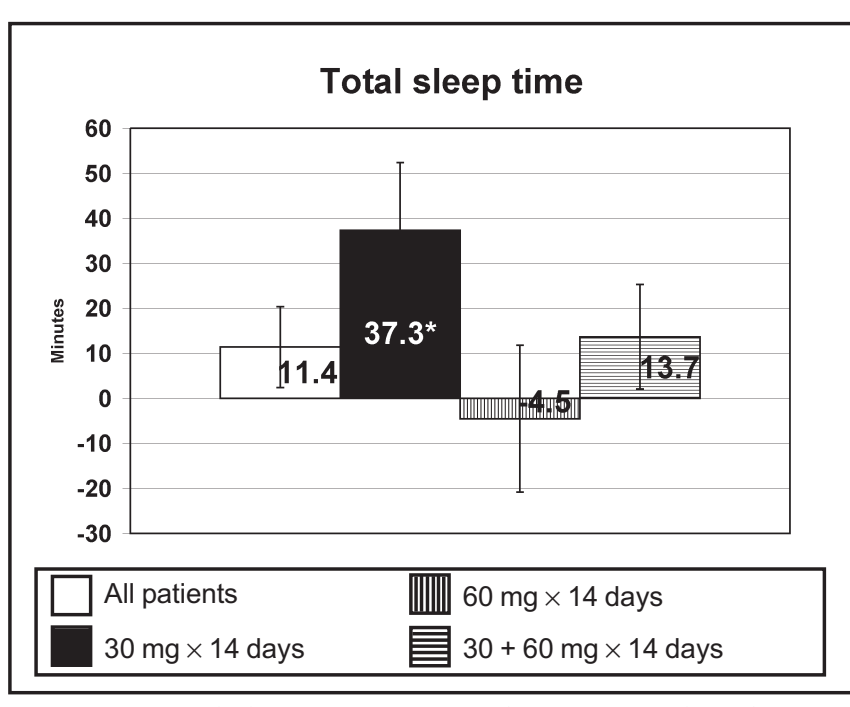

Figure 2. Total sleep time, mean change from baseline at day 14. Increase $=$ more time asleep; ${ }^{*} \mathbf{p}<0.05$.

series of questions in which participants rate their pain on a scale of 0 ("no pain") to 10 ("pain as bad as you can imagine"). Participants used a five-point scale to rate their acceptance of their current therapy. This scale was anchored at 0 , "none" and 4, "excellent." Finally, participants used a five-point scale anchored at 0 , "none" and 4 , "complete" to indicate the pain relief provided by their current therapy.

Neurocognitive battery. The neurocognitive battery was performed by a psychologist (SB) at screening (day 5), baseline (day 0), and during treatment (day 6, 15). A standardized neurocognitive battery was administered to participants. Alternative forms were employed where appropriate. The following cognitive domains with corresponding tests were administered: attention (Trails Making A, Digit Symbol Substitution Test: DSST), memory (Rey Auditory Verbal Learning Test, Immediate and Delayed Condition Recognition: RAVLT), motor-speed (Finger Tapping Speed Test), and executive function (Letter-Number Sequencing, Trails Making B).

Compliance. At each visit, participants were monitored for drug compliance and had to have taken at least 85 percent of the prescribed doses to be considered compliant. During the first week of treatment, if a participant missed $\geq$ one day of dosing or took $>$ seven capsules during any seven-day period, he or she was instructed on the proper dosing and the importance of maintaining the dosing schedule. If the participant continued to be noncompliant, he or she was dropped from the study.

\section{Safety evaluations}

Vital signs were recorded at screening and at each visit during treatment. A physical examination along with chemistry and hematology was performed at screening and prior to discharge at the last study visit. A 12-lead

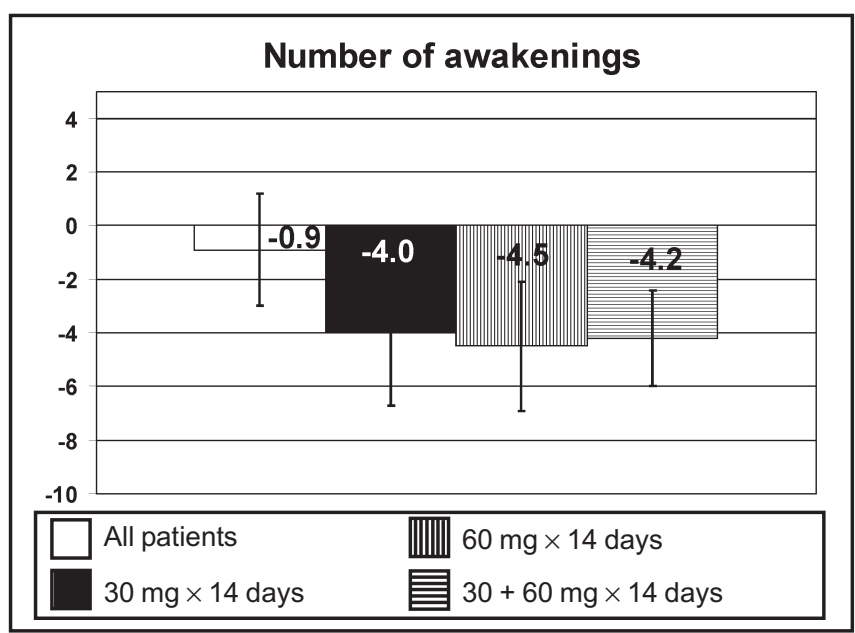

Figure 3. Number of awakenings, mean change from baseline at day 14 . Decrease = less awakenings.

ECG was also obtained at screening. Adverse event information was collected at each visit.

\section{Data analysis}

The efficacy endpoints were analyzed for compliant study participants who completed at least the day 6 evaluations (e.g., the evaluable population). The safety analysis was performed for all participants who were exposed to at least one dose of study medication. Parameters measured on more than one day (e.g., days 13 and day 14) during the screening, baseline, or treatment periods were defined as the average of the values obtained at each measurement period. Changes from baseline values were calculated both as a percent-change-from-baseline and as an absolute-change-from-baseline. Changes from screening were calculated as an absolute-change-frombaseline only. Nonparametric statistical methods (e.g., Wilcoxon tests) were used. Hypothesis testing was twosided and claims of significance were based on two-sided p-values of p-values of 0.05 or less.

\section{RESULTS}

\section{Demographics}

A total of 127 participants were screened. Of these, 93 did not progress to treatment because they failed the inclusion/exclusion criteria. No participant dropped out of the screening process because of inadequately controlled pain during the placebo washout period. Thus, 34 progressed to receive treatment (e.g., intent-to-treat population) and 31 participated for long enough to be considered evaluable. The demographics of the participants who completed the study include a mean age of 53.7 (range 26 to 75 ), majority female (27 [79 percent] vs 7 [21 percent]), majority Caucasian (21 [62 percent]), but with 
Table 1. PSG results

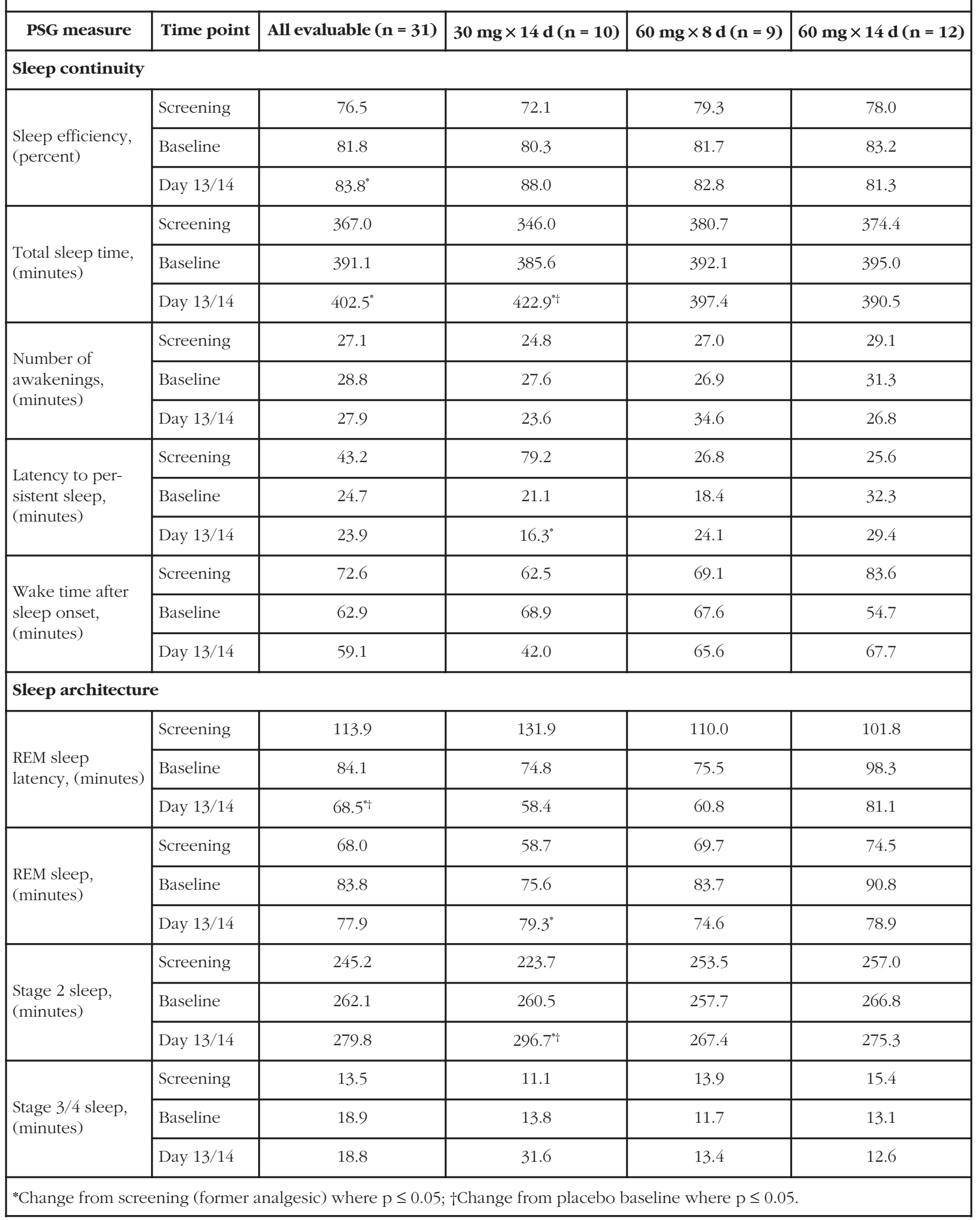


substantial African-American (10 [29 percent]) percentage and lesser percentages of Hispanic (2 [6 percent]) or other (1 $[3$ percent $])$.

Participants who received $30 \mathrm{mg} / \mathrm{d}$ of A-MQD for the entire study period will be referred to as the " $30 \mathrm{mg} \times 14$ day group," participants whose dosage was increased to $60 \mathrm{mg} / \mathrm{d}$ after the first six days of treatment will be referred to as the "60 $\mathrm{mg} \times 8$ day group," and participants whose dosage was increased to $60 \mathrm{mg} / \mathrm{d}$ and who continued with treatment for a full 14 days at this level will be referred to as the "60 $\mathrm{mg} \times 14$ day group." Day 13/14 and day 15 values refer to day $19 / 20$ and day 21 values, respectively, for this latter group.

As mentioned earlier, the interim analysis suggested that the study results for the $30 \mathrm{mg} \times 14$ day group and the $60 \mathrm{mg} \times 8$ day group would not represent comparable periods of uniform exposure to study drug. Thus, the rest of this document will largely focus on the results for the $30 \mathrm{mg} \times 14$ day group and the $60 \mathrm{mg} \times 14$ day group. The results for the $60 \mathrm{mg} \times 8$ day group are included in the appropriate tables, but will not be discussed further.

\section{Polysomnography}

Given the relatively small size of the study population, measures of sleep continuity initiation, maintenance, and architecture are presented for the participant population as a whole and as a function of dose groups (Table 1 and Figures 2 to 4 ).

Compared with normal sleepers, the sleep architecture of the study population at screening reflected their sleep disturbances (increased sleep stage 1 [data not shown]; diminished sleep stages 2 and 3/4; longer REM latency), even while stable on prior pain therapy. At baseline, subsequent to the withdrawal of all pain medications, some of these differences were attenuated suggesting that the participants' previous therapies were negatively affecting their sleep (Table 1). Overall, compared with baseline, treatment with A-MQD preserved sleep architecture and tended to increase stage 2 and stage 3/4 sleep and reduce REM latency.

At study end, REM sleep duration was slightly depressed, dropping to 77.9 minutes vs 83.8 minutes at placebo-baseline. This effect was largely due to a decrease of 11.0 minutes in the $60 \mathrm{mg} \times 14$ day group. In contrast, the $30 \mathrm{mg} \times 14$ day group saw an increase of 3.7 minutes of REM sleep over this period.

For the total study population, PSG sleep parameters were consistently improved by A-MQD compared with measures obtained at screening while on prior therapy. Both Total Sleep Time (TST) and Sleep Efficiency (SE) were significantly increased by study drug while Latency to Persistent Sleep (LPS) and Wake-time-after-SleepOnset (WASO) both trended downward (Table 1). However, although some trends did emerge, A-MQD did

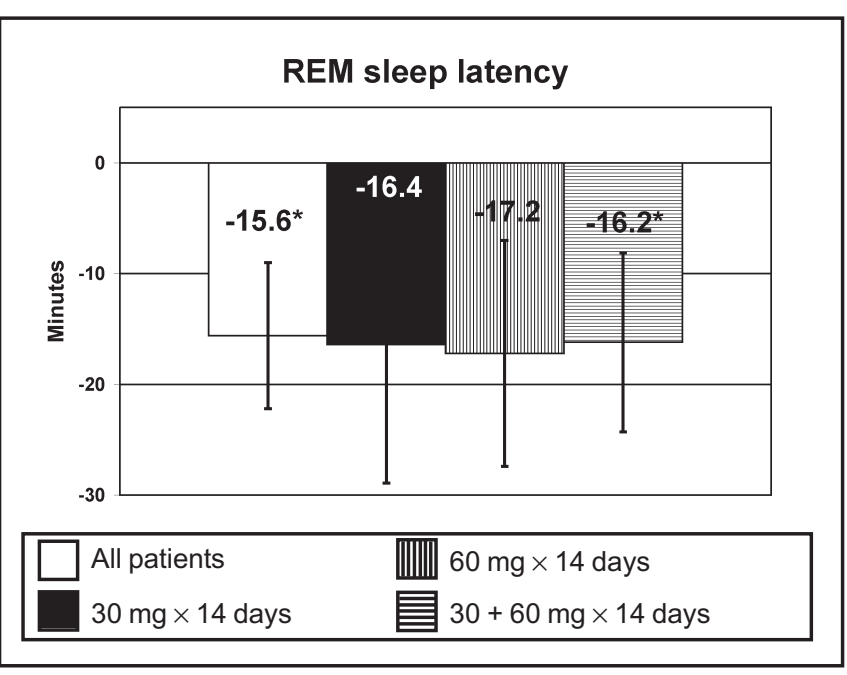

Figure 4. REM sleep latency, mean change from baseline at day 14. Decrease $=$ Less time to REM sleep; ${ }^{*} \mathbf{p}<0.05$.

not significantly impact PSG measures of sleep initiation and maintenance as compared with placebo baseline. Specifically, TST and SE increased while WASO and the Number of Awakenings (NAW) both decreased (Table 1).

The treatment subgroups demonstrated varying results on PSG outcome measures. The $30 \mathrm{mg} \times 14$ day group was consistently improved relative to placebo baseline in all aspects of sleep: LPS, WASO, NAW, and REM sleep latency were reduced while SE and TST were increased (Figures 2 to 4). Less consistent results for these measures were seen in $60 \mathrm{mg} \times 14$ day group.

\section{Subjective assessments}

Subjective participant assessments of nighttime sleep indicated significant improvement with A-MQD (Table 2 and Figure 5). The entire participant population showed significant improvements in subjective sleep quality at both day 6 and day 15 (p $=0.0001, p \leq 0.0001)$ relative to placebo-baseline values. Significance was maintained at day 6 for the $30 \mathrm{mg} \times 14$ day group $(\mathrm{p}=0.0254)$ and at day 15 for the $60 \mathrm{mg} \times 14$ day groups $(p=0.0010)$. The study population reported sleeping for a mean of $30 \mathrm{~min}-$ utes longer than at baseline at both the day $6(\mathrm{p}=0.0691)$ and day $15(\mathrm{p}=0.0286)$ time points. This increase in selfrated Number of Hours of Sleep was significant for the 30 $\mathrm{mg} \times 14$ day group (1.1 hours, $\mathrm{p}=0.0313)$ and approached significance for the $60 \mathrm{mg} \times 14$ day group (0.8 hours, $\mathrm{p}=0.0625)$ at day 15 .

Daytime alertness, as measured by the ESS, was impacted to a small, but significant degree for the study population as a whole at both day $6(\mathrm{p}=0.015)$ and day $15(\mathrm{p}=0.0150)($ Table 2$)$, but no treatment group showed significant reduction in alertness as compared with placebo baseline.

Participants' assessments of their pain severity and 
Table 2. Subjective measures of sleep

\begin{tabular}{|c|c|c|c|c|c|}
\hline $\begin{array}{l}\text { Subjective } \\
\text { measure }\end{array}$ & Time point & $\begin{array}{l}\text { All evaluable } \\
\quad(n=31)\end{array}$ & $\begin{array}{c}30 \mathrm{mg} \times 14 \mathrm{~d} \\
(\mathrm{n}=10)\end{array}$ & $\begin{array}{c}60 \mathrm{mg} \times 8 \mathrm{~d} \\
(\mathrm{n}=9)\end{array}$ & $\begin{array}{c}60 \mathrm{mg} \times 14 \mathrm{~d} \\
(\mathrm{n}=12)\end{array}$ \\
\hline \multirow{4}{*}{$\begin{array}{l}\text { Epworth sleepiness } \\
\text { score }(<8 \text {, normal; } \\
\text { higher score } \\
\text { indicates more } \\
\text { sleepiness) }\end{array}$} & Screening & 5.3 & 4.4 & 6.3 & 5.3 \\
\hline & Baseline & 4.7 & 3.7 & 5.3 & 5.0 \\
\hline & Day 6 & $5.4^{*}$ & 4.5 & 6.7 & 5.2 \\
\hline & Day 14 & $6.6^{*}$ & 5.4 & 7.5 & 6.8 \\
\hline \multirow{4}{*}{$\begin{array}{l}\text { Number of hours } \\
\text { of sleep }\end{array}$} & Screening & 5.9 & 5.9 & 5.7 & 6.0 \\
\hline & Baseline & 6.1 & 6.3 & 6.0 & 6.0 \\
\hline & Day 6 & 6.6 & 7.2 & 5.9 & 6.5 \\
\hline & Day 14 & $6.6^{* \dagger}$ & $7.4^{*+}$ & 5.3 & $6.8^{\dagger}$ \\
\hline \multirow{4}{*}{$\begin{array}{l}\text { Overall quality } \\
\text { of sleep }(0, \text { poor } \\
\text { sleep; } 100, \text { best } \\
\text { sleep; increase, } \\
\text { better sleep) }\end{array}$} & Screening & 33.3 & 27.3 & 35.9 & 36.3 \\
\hline & Baseline & 40.9 & 46.1 & 43.4 & 34.7 \\
\hline & Day 6 & $57.9^{*}$ & $64.7^{*}$ & $55.0^{*}$ & 54.5 \\
\hline & Day 14 & $64.3^{* \dagger}$ & $61.8^{\dagger}$ & $61.4^{*+\dagger}$ & $68.3^{* \dagger}$ \\
\hline
\end{tabular}

their acceptance of, and relief provided by, the study drug indicate that A-MQD was an effective treatment for osteoarthritis pain (Table 3). Relative to placebo-baseline values, the BPI Average Pain scores were significantly improved for the total population (day 6, $p=0.0016$; day $15, \mathrm{p} \leq 0.0001$ ), the $30 \mathrm{mg} \times 14$ day group (day $6, \mathrm{p}=$ 0.0098 ; day $15, \mathrm{p}=0.0078$ ) and the $60 \mathrm{mg} \times 14$ day group (day $15, \mathrm{p}=0.0039$ ) (Table 3 ). The study population as a whole showed a significant increase in Acceptance of Current Therapy at day $6(\mathrm{p}=0.0011)$ and day $15(\mathrm{p}=$ $0.0006)$ and indicated experiencing a significant improvement in Relief from Current Therapy (day 6 and day 15, p $\leq 0.001$ ) relative to placebo-baseline values.

\section{Neurocognitive battery}

Treatment with A-MQD significantly enhanced cognition as compared with performance on prior analgesics and after a wash-out phase. When all treatment groups were combined $(\mathrm{n}=31)$ significant improvement from screening (prior analgesics) was observed at day 15 for Letter Number Sequencing, Trails Making $\mathrm{A} \& \mathrm{~B}, \mathrm{DSST}$, and RAVLT Immediate and Delayed Condition tests $(\mathrm{p}=0.0237, \mathrm{p}=0.0004, \mathrm{p}=0.0039, \mathrm{p}=$ $0.0231, \mathrm{p}<0.0001$, and $\mathrm{p}<0.0001$, respectively). Trend improvement or no change was observed for other measures.
Significant improvements were also seen relative to placebo baseline on the RAVLT $(p=0.0136)$ and Trail Making part A $(\mathrm{p}=0.0045)$ evaluations. Trend improvement or no change was observed for other measures. A thorough presentation of these results will be reported elsewhere.

\section{Safety}

A-MQD was generally safe and well tolerated. Side effects did not appear to exhibit a dose effect. Twentytwo patients experienced one or more adverse events, most of which were mild to moderate in severity. The most common adverse events (occurring in $>10$ percent of all treated participants) were nausea $(n=10,29$ percent), sedation ( $n=5,15$ percent), constipation ( $n=5,15$ percent), vomiting ( $\mathrm{n}=4,12$ percent), and pruritus $(\mathrm{n}=$ 4, 12 percent). All of these events were consistent with the known effects of the study drug and had been observed in previous clinical trials.

Serious adverse events (SAEs) occurred in one participant (3 percent) while receiving $60 \mathrm{mg} / \mathrm{d}$ for eight days. The SAEs (experienced simultaneously) were severe sedation and unresponsiveness in this participant who was subsequently found to have previously undiagnosed hypothyroidism. The participant subsequently recovered from these adverse events. 
Table 3. Subjective measures of pain therapy

\begin{tabular}{|c|c|c|c|c|c|}
\hline $\begin{array}{c}\text { Subjective } \\
\text { measure }\end{array}$ & Time point & $\begin{array}{l}\text { All evaluable } \\
\quad(n=31)\end{array}$ & $\begin{array}{c}30 \mathrm{mg} \times 14 \mathrm{~d} \\
(\mathrm{n}=10)\end{array}$ & $\begin{array}{c}60 \mathrm{mg} \times 8 \mathrm{~d} \\
(\mathrm{n}=9)\end{array}$ & $\begin{array}{c}60 \mathrm{mg} \times 14 \mathrm{~d} \\
(\mathrm{n}=12)\end{array}$ \\
\hline \multirow{4}{*}{$\begin{array}{l}\text { BPI: "Pain on } \\
\text { Average" }(0, \text { no } \\
\text { pain; 10, worst } \\
\text { pain) }\end{array}$} & Screening & 6.1 & 6.6 & 5.3 & 6.3 \\
\hline & Baseline & 6.1 & 6.2 & 5.2 & 6.7 \\
\hline & Day 6 & 5.3 & 4.5 & 4.9 & 4.9 \\
\hline & Day 14 & $4.1^{* \dagger}$ & $3.8^{*+t}$ & 4.1 & $4.3^{*+}$ \\
\hline \multirow{4}{*}{$\begin{array}{l}\text { Acceptability } \\
\text { of therapy } \\
\text { ( } 0 \text {, none; } \\
5 \text {, excellent) }\end{array}$} & Screening & 2.5 & 2.4 & 2.6 & 2.5 \\
\hline & Baseline & 2.7 & 3.0 & 3.1 & 2.3 \\
\hline & Day 6 & 3.6 & 3.7 & 3.4 & 3.6 \\
\hline & Day 14 & $3.8+$ & 3.7 & 4.0 & $3.8^{\dagger}$ \\
\hline \multirow{4}{*}{$\begin{array}{l}\text { Pain relief from } \\
\text { current } \mathrm{Rx} \\
(0, \text { none; } \\
5 \text {, excellent) }\end{array}$} & Screening & 2.4 & 2.3 & 2.3 & 2.4 \\
\hline & Baseline & 2.0 & 2.5 & 1.8 & 1.7 \\
\hline & Day 6 & 3.0 & 3.2 & 2.6 & 3.1 \\
\hline & Day 14 & $3.5^{*+}$ & $3.6^{\dagger}$ & $3.4^{*}+$ & $3.5^{*+}$ \\
\hline
\end{tabular}

\section{DISCUSSION}

This single-center, placebo-baseline-controlled, single-treatment, single-blind study was designed to evaluate the impact of using A-MQD on objective and subjective measures of sleep as well as pain in participants with osteoarthritis complaining of sleep disturbances. Objective single-blind placebo baseline sleep characteristics confirmed the subjective perception of sleep disturbance due to chronic pain. The sleep fragmentation, low sleep efficiency, and poor subjective sleep quality in these chronic osteoarthritis participants were consistent with observations in other sleep studies in chronic pain participants. ${ }^{16-18}$ Importantly, these sleep disturbances were present to an even greater degree during screening when participants were observed on their previous stable regimens of pain and sleep aids.

After withdrawal of all pain and sleep medication to establish baseline characteristics, participants were treated with either 30 or $60 \mathrm{mg} / \mathrm{d}$ of study drug for a period of 14 to 20 days. The results were very encouraging and intriguing: on the basis of objective and subjective outcome measures, the study drug improved both sleep and pain. Although improvements were not always statistically significant, likely owing to the small sample size, the overall trends were positive.

Treatment effects on sleep architecture included increases in the amount stage 2 and stage 3/4 sleep relative to both placebo baseline and screening (prior therapy) levels. In contrast to previous PSG studies of acute dosing with opioids at bedtime, A-MQD increased NREM sleep time when taken in the morning. REM latency was also significantly reduced with respect to both placebo baseline and screening observations. While minor, nonsignificant REM suppression vs baseline was seen with AMQD, the time spent in REM sleep was markedly increased relative to the values seen at screening when participants were still taking their prior pain medication.

In addition, objective measures of sleep initiation and continuity as assessed in the participant population as a whole were improved by study drug when compared with placebo-baseline levels. TST and SE were increased while LPS, NAW, and WASO were reduced. Importantly, LPS, SE, TST, and WASO improvements were even greater when compared with screening values obtained when participants were on their previous stable regimens of pain and sleep medications.

Subjective assessments of sleep quality indicated that 


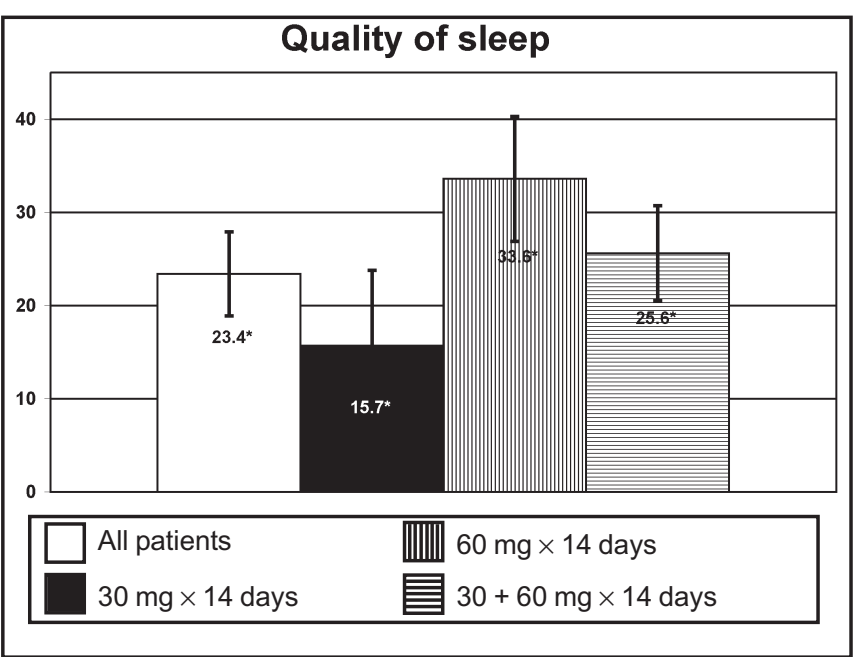

Figure 5. Quality of sleep, mean change from baseline at day 14. Increase = Better sleep; Scale: $0=$ Poor sleep; $100=$ Best sleep* $\mathbf{p}<0.05$.

there was a clear perception of improved sleep with AMQD. Perceived sleep quality and the subjective Number of Hours of Sleep were significantly improved across the total participant population. Again, these improvements were even greater when compared with screening (prior therapy) levels. Although daytime sleepiness as measured by ESS was significantly impaired relative to placebo baseline for the total population at both the day 6 and day 15 , the actual ESS scores were within the range traditionally considered normal (ESS $<7$ ). In view of the well-known sedative effects of morphine, it is reasonable to observe some drowsiness in this relatively short study with its limited time to tolerate this effect. Study results may indicate that A-MQD's extended release formulation may produce milder effects on alertness than an immediate release treatment.

A-MQD was also clearly effective as a pain medication. Significant improvements were seen at both days 6 and 15 on the BPI and participant ratings of the Acceptance of and Relieffrom Current Therapy. Scores on each of these scales also indicated improvements relative to screening values, indicating that the study drug was better at managing pain symptoms than the previous therapies utilized by participants.

Furthermore, results from the neurocognitive battery seemed to indicate that A-MQD may have enhanced cognition in this participant group relative to prior therapy. While the underlying biological underpinnings of these findings are unknown, it is possible that either the reduction of pain or the resumption of sleep may have accounted for the enhancement of mnemonic and attentional functioning. These results will be presented at greater length in a subsequent publication.

As mentioned earlier, a limitation of this study is its design as a placebo-baseline-controlled, single-treatment, single-blind study. However, the choice of a baseline control design was deliberate because of two concerns. First, there were significant ethical concerns that a separate placebo control arm design would potentially convey negative consequences to participants resulting from the cessation of pain relief therapy. Second, concerns about loss of study sensitivity linked to the expected heterogeneity of the study population were expected. These expectations of participant heterogeneity were supported by the need to increase the dose of study medication for those participants complaining of inadequate pain relief at day 6 . We hypothesize that participants in the 30-mg group had mild to moderate chronic osteoarthritic pain, while participants who received $60 \mathrm{mg}$ for any period of time could be considered to have moderate to severe pain, which was more difficult to control by the use of medication. This difference in pain level could be expected to significantly impact the variability of improvements in sleep seen across treatment groups, especially if the dose level did not reach therapeutic levels for an individual participant.

The extension of time under treatment for participants in the $60 \mathrm{mg} \times 14$ day group added a temporal difference into study group comparisons. This change was introduced to allow the acute effects associated with transition to a new dose level to stabilize and permit equal amounts of time at a pain relieving dose. Nonetheless, it may be argued that the drug effect after two weeks (observed for participants in the $30 \mathrm{mg} \times 14$ day and $60 \mathrm{mg} \times 8$ day groups) may not be comparable to the drug effect seen after three weeks of treatment (60 $\mathrm{mg} \times 14$ day group). Morphine therapies are associated with the evolution of some level of tolerance to side effects, so the protocol change was expected to make the groups more comparable.

The results of this study suggest that A-MQD may be an effective treatment for participants with osteoarthritis pain and accompanying sleep disturbances. The improvements seen in all participants within measures of pain and sleep relative to untreated participants (placebo baseline) and participants with previous pain therapy (screening) may indicate that the previous therapies 1) were insufficient for the level of pain being experienced and 2) may have exacerbated the secondary sleeping difficulties experienced by participants. These data confirm the activity of A-MQD for the treatment of chronic moderate to severe osteoarthritis pain and support prior observations that with A-MQD pain therapy participants may expect improvements in the sleep disturbances commonly experienced by this participant population. More research could provide additional, helpful insights to inform clinicians' choices regarding the relationship between management of chronic pain and its associated sleep disturbances as well as further delineate A-MQD's ability to improve sleep and daytime functional outcomes in chronic pain populations. 


\section{ACKNOWLEDGMENTS}

The authors gratefully acknowledge the technical contributions of Cheryl Spinweber, PhD, and Al Agbulos, RPSGT, in reviewing the polysomnograms in this research study.

Murray Rosenthal, DO, California Clinical Trials, San Diego, California.

Polly Moore, PhD, California Clinical Trials, San Diego, California.

Eric Groves, MD, PhD, Ligand Pharmaceuticals Inc, San Diego, California.

Tad Iwan, BA, Ligand Pharmaceuticals Inc, San Diego, California.

Lisa Greenberg Schlosser, MS, Ligand Pharmaceuticals Inc, San Diego, California.

Zofia Dziewanowska, MD, PhD, Ligand Pharmaceuticals Inc, San Diego, California.

Andres Negro-Vilar, MD, PhD, Ligand Pharmaceuticals Inc, San Diego, California.

\section{REFERENCES}

1. Benca RM, Ancoli-Israel A, Moldofsky H: Special considerations in insomnia diagnosis and management: Depressed, elderly and chronic pain populations. J Clin Psychiarty. 2004; 65 (Suppl 8): 26-35.

2. Sutton DA, Moldofsky H, Badley EM: Insomnia and health problems in Canadians. Sleep. 2001; 24: 665-670.

3. McCracken LM, Iverson GL: Disrupted sleep patterns and daily functioning in patients with chronic pain. Pain Res Manag. 2002; 7: 75-79.

4. Nicassio PM, Wallston KA: Longitudinal relationships among pain, sleep problems and depression in rheumatoid arthritis. $J$ Abnorm Psychol. 1992; 101: 514-520.

5. Drewes AM, Jannum P, Andreasen A, et al.: Self-reported sleep disturbances and daytime complaints in women with fibromyalgia and rheumatoid arthritis. J Musculoskeletal Pain. 1994; 2: 15-31.

6. Moffitt PF, Kalucy EC, Kalucy RS, et al.: Sleep difficulties, pain and other correlates. J Intern Med. 1991; 230: 245-249.

7. Moldofsky H, Scarisbrick P, England R, et al.: Musculosketal symptoms and non-REM sleep disturbance in patients with "fibrositis syndrome" and healthy subjects. Psychosom Med. 1975; 37: 341-351.

8. Moldofsky H, Scarisbrick P: Induction of neurasthenic musculoskeletal pain syndrome by selective sleep stage deprivation. Psychosom Med. 1976; 38: 35-44.

9. Drewes AM, Nielson KD, Arendt-Nielsen L, et al.: The effect of cutaneous and deep pain on the electroencephalogram during sleep: An experimental study. Sleep. 1997; 20: 632-640.

10. Kay DC, Einstein RB, Jasinski DR: Morphine effects on human REM state, waking state and NREM sleep. Psychopharmacologica. 1969; 14: 404-416.

11. Caldwell JR, Rapport RJ, Davis JC, et al.: Efficacy and safety of once daily morphine formulation in chronic, moderate to severe osteoarthritis pain. J Pain Symptom Manage. 2002; 23(4): 278-291.

12. Rauck RL, Bookbinder SA, Bunker TR, et al.: The ACTION study: A randomized, open-label, multicenter trial comparing SID ER morphine sulfate capsules (AVINZA ${ }^{\circledR}$ ) to BID controlled-release oxycodone hydrochloride tablets (OxyContin ${ }^{\circledR}$ ) for the RX of chronic, moderate to severe low back pain. $J$ Opioid Manage. 2006; 2(3): 155-166.

13. Panjabi SS, Panjabi RS, Lawson KL, et al.: Cognitive function in chronic pain patients treated with once-a-day AVINZA $^{\circledR}$ (morphine sulfate extended-release capsules). In the 22nd Annual Meeting of the American Academy of Pain Medicine, February 22-25, 2006. San Diego CA: American Academy of Pain Medicine, 2006: 83.

14. Panjabi SS, Panjabi RS, Lawson KL, et al.: Pain and emotional functioning assessments in patients treated with once-a-day AVINZA $^{\circledR}$ in the management of chronic non-malignant pain. In the 22nd Annual Meeting of the American Academy of Pain Medicine, February 22-25, 2006. San Diego CA: American Academy of Pain Medicine, 2006: 82-83.

15. Rechtschaffen A, Kales A: A Manual of Standardized Terminology: Techniques and Scoring System for Sleep Stages of Human Subjects. Los Angeles: UCLA Brain Information Service/Brain Research Institute, 1968.

16. Moldofsky H, Scarisbrick P, England P, et al.: Musculoskeletal symptoms and non-REM sleep disturbance in patients with "fibrositis syndrome" and healthy subjects. Psychosom Med. 1975; 37: 341-51.

17. Pilowsky I, Crettenden I, Townley M: Sleep disturbance in pain clinic patients. Pain. 1985; 23: 27-33.

18. Leigh TH, Hindmarch I, Bird HA, et al.: Comparison of sleep in osteoarthritic patients and age and sex matched controls. Ann Rheum Dis. 1988; 47: 40-42. 